\title{
A Bottom-Up Approach to Encouraging Sustained User Adoption of a Secure Text Messaging Application
}

\author{
Surafel Tsega ${ }^{1}$ Angeli Kalra ${ }^{2}$ Cesar T. Sevilla ${ }^{3}$ Hyung J. Cho ${ }^{4}$ \\ ${ }^{1}$ The Mount Sinai Hospital, Icahn School of Medicine at Mount Sinai \\ Hospital, New York, New York, United States \\ 2 New York, United States \\ 3 Population Health and Payer Systems, Mount Sinai Health System, \\ New York, New York, United States \\ ${ }^{4}$ Department of Quality, New York City Health and Hospitals, New \\ York, New York, United States \\ Appl Clin Inform 2019;10:326-330.
}

\begin{abstract}
Address for correspondence Surafel Tsega, MD, The Mount Sinai Hospital, Icahn School of Medicine at Mount Sinai Hospital, 1 Gustave L. Levy Place, Box 1086, New York, New York 10029, United States (e-mail: surafel.tsega@mountsinai.org).
\end{abstract}

\begin{abstract}
Keywords

- hospital communication systems

- secure text messaging

- smartphone

- confidentiality

- mobile applications

Background Inpatient providers are increasingly utilizing alternative communication modalities outside what has traditionally been used, including short messaging service text messaging and application-based chat tools. Text messaging that meets the recommendations of the Joint Commission ("secure text messaging") allows for the communication of sensitive patient information through an encrypted platform.

Objective In this quality initiative utilizing the Plan-Do-Study-Act (PDSA) model, we attempted two rollout designs to maximize user adoption of a secure text messaging application.

Methods Our institution launched a secure text messaging application (Cureatr) using a top-down approach during the first PDSA cycle, defined as communication and outreach through department chairs and administrative leaders throughout the hospital. After inadequate user adoption, we transitioned to a bottom-up approach in the second PDSA cycle, defined as direct communication and engagement with end users. This campaign targeted the hospital medicine and inpatient social work department, and used discharge planning as a use case to encourage adoption.

Results Over a 6-month period, we observed an increase in active users in the hospital medicine department ( 7.5 unique users per month to 29 users during the first and second PDSA cycles, $p<0.01)$. Additionally, we saw an increase in messages sent and received by the medicine (687 messages on average per month and 7,367 messages per month in each respective PDSA cycle, $p<0.01)$ and social work departments (350 messages on average per month and 6,083 messages during the first and second PDSA cycle, $p<0.01$ ). We did not observe a significant change in active users or messages sent in the surgery department, which did not participate in the second PDSA cycle.

Conclusion We believe that a bottom-up approach is important to introducing mobile applications to the inpatient setting and can contribute to sustained user adoption.
\end{abstract}

received

January 18, 2019

accepted after revision

March 5, 2019 (c) 2019 Georg Thieme Verlag KG Stuttgart · New York
DOI https://doi.org/

10.1055/s-0039-1688554.

ISSN 1869-0327. 


\section{Background and Significance}

Inpatient clinical providers are increasingly utilizing alternative communication modalities outside what has traditionally been used. Whereas providers previously communicated by phone and numerical text paging, they are now turning to short messaging service (SMS) text messaging and application-based chat tools to communicate within their team and across multidisciplinary groups., ${ }^{1,2}$ Within this context, SMS texting is oftentimes preferred over traditional methods in clinical settings. ${ }^{3}$ As a result of this, there is increasing concern about communicating sensitive patient information within the confines of the Health Insurance and Portability Accountability Act (HIPAA) and recommendations laid out by the Joint Commission. ${ }^{1,4}$

As Drolet et al explain-HIPAA is technologically agnostic and does not specifically comment on communication through text messaging platforms. Instead, the Joint Commission has made attempts to identify guidelines for "secure text messaging" that allows for the communication of sensitive patient information through an encrypted platform (notably, texting physician orders is prohibited). - Table 1 outlines the recommendations laid out by the Joint Commission. ${ }^{5}$ Despite these recommendations, widespread implementation of secure text messaging is not the current reality. A recent study revealed that $80 \%$ of clinicians are still issued pagers by their hospital. ${ }^{6}$ Additionally, over $85 \%$ of physicians in training programs were using smartphones in the clinical setting, but only a quarter reported their organization had implemented a secure text messaging platform, and only $7 \%$ admit that it was what most clinicians in their organization were using. ${ }^{7}$ Successful efforts from the use of applicationbased chat tools (such as WhatsApp) are promising, as they are user-friendly and already well-adopted by the public. ${ }^{8,9}$ However, applications that may be assumed to be secure may not necessarily achieve patient confidentiality without additional safeguards by the end user. ${ }^{10}$

Several early studies have demonstrated the successful adoption of various secure text messaging applications. While this evidence also shows that it is more favorable and improves communication compared to traditional paging systems, there is a paucity of studies demonstrating how to best implement this communication application. ${ }^{11-13}$ Moreover, the efforts cited thus far were tried in smaller settings (four units or less).

Our institution identified secure text messaging as an important tool that should be available for its providers. The leadership acknowledged the prevalence of SMS communication and hoped that this application would bring providers'

Table 1 Key features defining a secure text messaging platform as outlined by the Joint Commission ${ }^{5}$
- Secure sign-on process
- Encrypted messaging
- Delivery and read receipts
- Date and time stamp
- Customized message retention time frames

actions in line with the Joint Commission recommendations without prohibiting necessary communication. With this operational mandate, we set out to effectively introduce a secure text messaging platform across the institution, and in the process identified one strategy that proved to be more effective than the other.

\section{Methods}

Our initiative was introduced at The Mount Sinai Hospital, a 1,134-bed tertiary urban academic hospital in New York City. The study period was approximately 13 months, from June 13, 2017 to July 31, 2018. We utilized Cureatr, a secure text messaging application that meets the recommendations laid out by the Joint Commission. The application was introduced as a means of communication to replace noncompliant SMS texting and was meant to augment but not replace standard paging practices between providers. The application has both a mobile and desktop platform. All providers (attending physicians, house staff, nurse practitioners, and physician assistants) were provided with an account at the beginning of the initiative. Users downloaded the application from the appropriate App Store and used single sign-on functionality to log in using their institutional credentials. Users could message any provider with a provisioned account, and would receive a read receipt when a message was received. Providers that had not previously logged into their account were sent an automated email directing them to pending messages in the application. Users were also notified if a provider was not active within the application. We utilized Plan-Do-Study-Act (PDSA) cycles as our guide for assessing success of the project, defined loosely as sustained user adoption of the platform.

The initial rollout strategy was a major hospital-wide rollout among all providers in the inpatient setting. This campaign was defined by a top-down approach, which included hospitalwide email communications; dissemination of information through residency and fellowship program directors; dissemination across clinical departments through department chairs; and presentations at departmental grand rounds to the largest departments (including surgery and internal medicine). The application was introduced to the inpatient social work department through department leadership communication. A go-live date was set and email reminders were sent to clinicians leading up to the event. Active users were defined as any user who sent or read a message during the defined period; messages sent by users in each department were tracked as well. Usage data was monitored during this process. Several months after launching this campaign, we realized adoption was not meeting expected targets. We debated the current rollout plan and adjusted our approach.

Though this project achieved considerable institutional support and was messaged from the upper hospital administration and departmental leadership to the frontline users, we decided that a bottom-up approach may be more effective for adoption. During this second rollout campaign (also referred to as the second PDSA cycle), we targeted two specific departments, internal medicine (historically an early adopter of 
initiatives at our institution) and social work, through targeted email communications, in-person announcements during interdisciplinary rounds, and the identification of one department champion to promote the application. This champion utilized direct communication with users (in-person and through email) and identified and resolved any outstanding barriers to user adoption. We also identified a use case at the request of the social work department-discharge planningas a means of encouraging utilization. While no structured discharge planning workflow existed, providers and social workers were encouraged to use the group chatting feature to communicate any discharge planning needs with the interdisciplinary team (which also included the unit medical director and case manager). This bottom-up approach also included more active communication after campaign launch, focusing on user adoption and engagement. We compared usage data for the internal medicine and social work departments with the surgical department, which did not receive the bottom-up approach. Usage data was obtained through the vendor. Subsequent statistical analysis was done using a twosample $t$-test. Though the campaign began as an operational directive from the hospital administration, the project was subsequently reviewed by the Quality Improvement Committee in the Department of Medicine at Mount Sinai Hospital, and was deemed a quality improvement project.

\section{Results}

Our study period is broken into the initial large-scale rollout campaign (June 2017-January 2018), and the second PDSA cycle, defined as the second rollout campaign (February 2018-July 2018). A total of 1,346 inpatient providers were provisioned at the start of the initiative. Of note, technical issues were identified soon after the initial rollout campaign, involving incorrect credentialing of users and the inability to log in; these issues were resolved by August 2017. Outreach in the form of hospital-wide communication, direct communication with users, presentations to department leaders, and previously planned presentations at departmental grand rounds continued during and after this time period. It is also important to note that the social work department did not join the secure text messaging platform until October 2017 (when 632 users were provisioned).

-Fig. 1 shows the active monthly users. During the initial rollout campaign, the internal medicine department averaged 7.5 unique users per month (out of 36 potential users on service at one time); this increased to 29 users per month during the second PDSA cycle $(p<0.01)$. The social work department averaged 44.5 users per month following the initial rollout campaign and 83 users per month during the second campaign (47 potential inpatient users were expected on service at one time; $p=0.286$ ). Of note, the increase in active users seen in the social work department in November 2017 and March 2018 represent efforts to provision and activate new users in the social work department. The surgery department averaged 9.5 users during the first campaign and 14 users during the second campaign (out of a total of 61 potential users on service at one time; $p=0.544$ ).

-Fig. 2 shows the monthly messages sent by each department. In the internal medicine department, 687 messages were sent on average per month in the preintervention period and 7,367 messages per month in the postintervention period $(p<0.01)$. In the social work department, 350 messages were sent on average per month in the preintervention phase and 6,083 messages were sent postintervention $(p<0.01)$. In the surgery department, an average of 44 messages were sent preintervention, and 89 messages were sent postintervention ( $p=0.209)$.

\section{Discussion}

Our experience suggests that a targeted rollout campaign that utilizes a bottom-up approach can be a more effective strategy to improving user adoption of novel communication

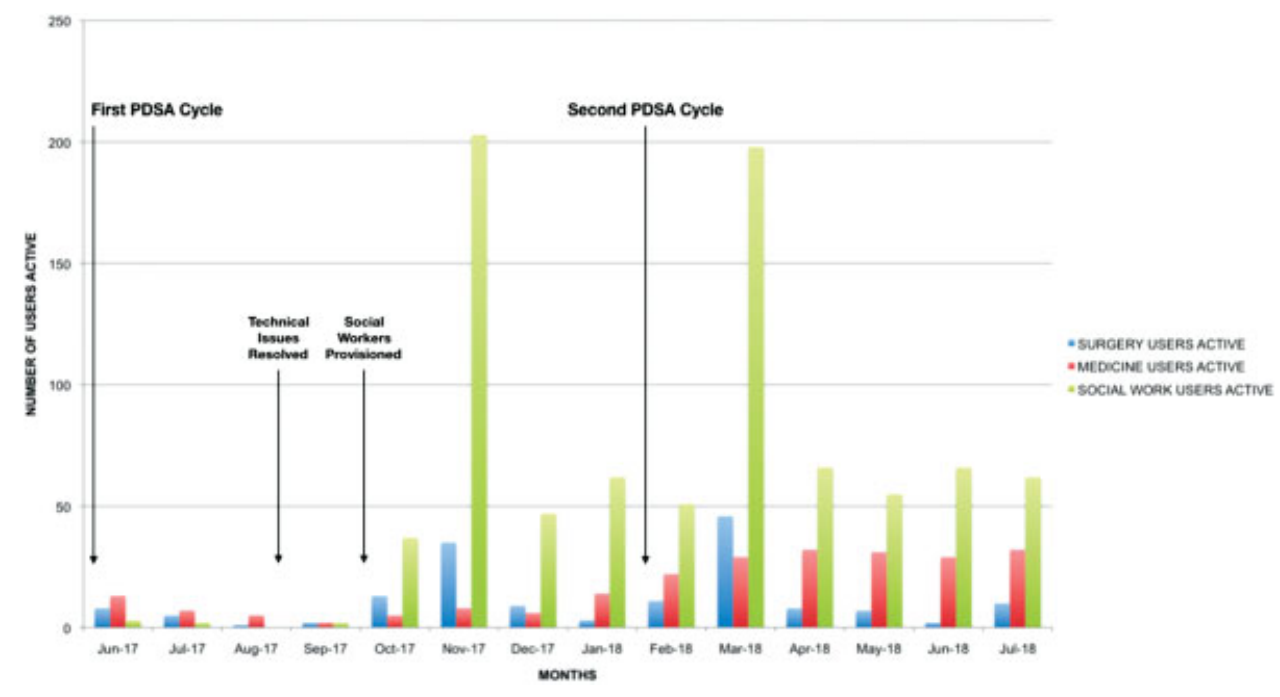

Fig. 1 Active users per department per month. Active users were defined as any user who sent or read a message during the defined period. Of note, the increase in active users seen in the social work department in November 2017 and March 2018 represent efforts to batch provision and activate new users in the social work department. PDSA, Plan-Do-Study-Act. 


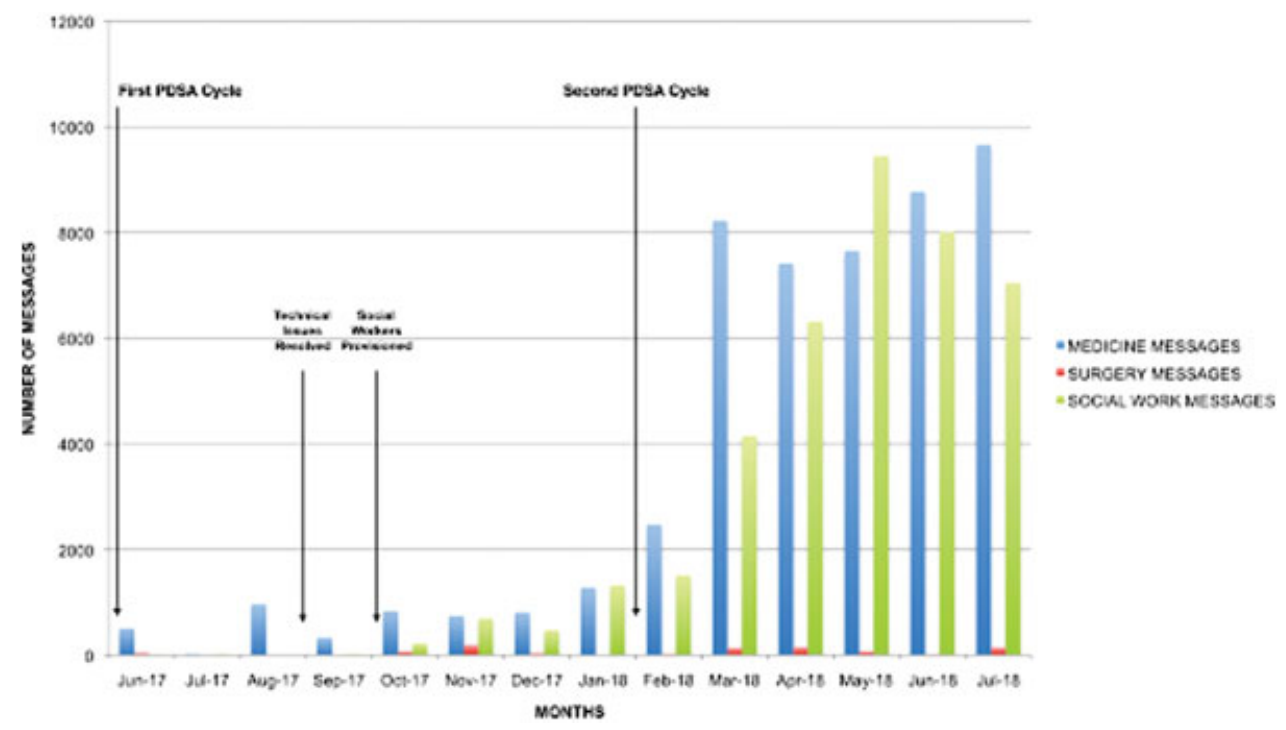

Fig. 2 Messages sent by each department per month. PDSA, Plan-Do-Study-Act.

tools in the inpatient setting. Identifying important use cases to introduce the tool into current workflows is also important for widespread adoption.

The idea of a bottom-up approach to introducing important initiatives has been established in the quality improvement literature. ${ }^{14,15}$ Haun et al, who conducted a survey of care teams using secure messaging with patients, described a culture change that involved proactively educating staff, promoting use of secure messaging, and actively integrating it into the current workflow. ${ }^{16}$ Both approaches resulted in widespread and sustained adoption of the respective initiatives similar to what we experienced.

We believe that the targeted rollout campaign ultimately led to increased adoption and sustained utilization as a result of frequent, personalized outreach to the departments and users through individual in-app messaging and in-person contact, along with frequent announcements during interdisciplinary rounds. Identifying a use case was also integral to sustained adoption as it gave a clear reason for individuals to use the application (to more easily coordinate discharge planning), even in the absence of a structured workflow. Importantly, the increase seen also represents persistent use-inpatient treatment teams rotate on and off service every 2 to 4 weeks. Our results suggest that as new users joined the rotation, they adopted the preferred mode of communication. Though labor intensive, this strategy is necessary to overcome the barriers we highlight, thereby dramatically changing provider practices.

We believe that the poor rates of adoption early may also have been due in part to inertia or resistance to changing the entrenched culture around communication. Users may not have been incentivized to use the application as there were no perceived disadvantages to using established modes of communication. This phenomenon has been previously described by Prochaska et al, who surveyed internal medicine residents. Respondents identified SMS text messaging as their preferred method of communication despite the security concerns because of the perceived benefit in coordination and delivery of care. ${ }^{4}$ Requiring users to download and use a new application can be a considerable barrier to deter widespread and sustained adoption. Additionally, the secure messaging platform was not integrated within the current electronic medical record system, and had to be introduced to well-established workflows, further burdening providers.

Our targeted campaign also identified another important issue worth considering when introducing a secure text messaging application. At our institution, the application is currently not available to nurses. As a result, standard text paging remains the standard for communication with nurses and the ancillary services. Providers were asked to manage multiple lines of communication through several platforms. The addition of a secure messaging application (in addition to standard communication modalities) created additional disruptions to the current workflow that providers had to adjust to.

Analysis of our data does include some limitations. The initial rollout was hampered by technical issues for an unidentified amount of users, which could have contributed to lower user adoption. However, the outreach campaign continued during and after this time and was accompanied by additional email communication with users, which we believe limited the extent of the effects of these technical issues. Second, the social work department includes individuals in the inpatient and outpatient setting. While our outreach focused on those in the inpatient setting, we are unable to separate data by the clinical setting. We assume that the increased activity is from the inpatient social workers as there was no concerted effort to reach out to outpatient social workers, nor was there a new initiative during the intervention that targeted the outpatient group, meaning usage was unlikely to have been driven by increased activity by the outpatient group. Though we did not account for provider workload (e.g., patient census) or service workflow, our intention was to track the change in activity within a department, not between departments. Most importantly, while we were able to track usage within the application, we did not track communication 
outside the application (through SMS texting and standard numeric paging) to identify a shift in communication from one platform to another, or to quantify the percentage of communication that occurred within and outside the application.

In conclusion, a targeted, bottom-up approach to introducing a secure text messaging application is a reasonable option to consider when designing a rollout strategy. We believe that as hospitals take the necessary steps to adopt secure text messaging strategies, they should utilize an outreach strategy that is similarly personalized, multimodal, and sustained.

\section{Clinical Relevance Statement}

We believe that a bottom-up approach that identifies key frontline stakeholders and use cases is important to introducing mobile applications to the inpatient setting, particularly those that need to be introduced into established workflows, or are otherwise not easily integrated into existing technology systems.

\section{Multiple Choice Questions}

1. Your institution is planning to launch a new mobile application for providers to communicate securely with one another. You have been tasked with identifying a successful rollout campaign for the initiative. Which of the following campaign strategies has been shown to improve user adoption of a particular intervention?

a. E-mail communication sent by the Chief Medical Officer introducing a new mobile application.

b. E-mail communication sent by department chairs introducing a new mobile application.

c. E-mail communication targeting end users, utilizing division champions, and identifying a use case to promote adoption of a new mobile application.

d. Word-of-mouth promotion of a new mobile application.

Correct Answer: The correct answer is option c. The success of bottom-up approaches to introducing an initiative has been proven to be effective in the quality improvement literature. We showed that employing a similar method can result in sustained user adoption of a mobile application, particularly when introducing the application to pre-established workflows.

2. Which of the following features is considered to be an important part of a secure text messaging platform as outlined by the Joint Commission?

a. Secure sign-on process.

b. Date and time stamp.

c. Delivery and read receipts.

d. Encrypted messaging.

e. All of the above.

Correct Answer: The correct answer is option e. The Joint Commission defines a secure text messaging application as one that includes: (1) a secure sign-on process, (2) date and time stamp, (3) delivery and read receipts, (4) encrypted messaging, and (5) customized retention time frames.

Protection of Human and Animal Subjects

Our project was deemed a quality project by the Quality Improvement Committee in the Department of Medicine at Mount Sinai Hospital, and thus an Institutional Review Board submission was not required.

Conflict of Interest

None declared.

\section{References}

1 Drolet BC. Text messaging and protected health information: what is permitted? JAMA 2017;317(23):2369-2370

2 Frizzell JD, Ahmed B. Text messaging versus paging: new technology for the next generation. J Am Coll Cardiol 2014;64(24):2703-2705

3 Shah DR, Galante JM, Bold RJ, Canter RJ, Martinez SR. Text messaging among residents and faculty in a university general surgery residency program: prevalence, purpose, and patient care. J Surg Educ 2013;70(06):826-834

4 Prochaska MT, Bird AN, Chadaga A, Arora VM. Resident use of text messaging for patient care: ease of use or breach of privacy? JMIR Med Inform 2015;3(04):e37

5 "Standards FAQDetails: Texting - Use of Secure Text Messaging for Patient Information." Available at: https://www.jointcommission. org/standards_information/jcfaqdetails.aspx?StandardsFAQId= $1615 \&$ StandardsFAQChapterId $=8 \&$ ProgramId $=0$ \&ChapterId $=0 \&$ IsFeatured=False\&IsNew=False\&Keyword=text\%20messaging. Accessed February 11, 2019

6 O'Leary KJ, Liebovitz DM, Wu RC, et al. Hospital-based clinicians' use of technology for patient care-related communication: a national survey. J Hosp Med 2017;12(07):530-535

7 Franko OI, Tirrell TF. Smartphone app use among medical providers in ACGME training programs. J Med Syst 2012;36(05):3135-3139

8 Astarcioglu MA, Sen T, Kilit C, et al. Time-to-reperfusion in STEMI undergoing interhospital transfer using smartphone and WhatsApp messenger. Am J Emerg Med 2015;33(10):1382-1384

9 Johnston MJ, King D, Arora S, et al. Smartphones let surgeons know WhatsApp: an analysis of communication in emergency surgical teams. Am J Surg 2015;209(01):45-51

10 Drake TM, Claireaux HA, Khatri C, Chapman SJ. WhatsApp with patient data transmitted via instant messaging? Am J Surg 2016; 211(01):300-301

11 Przybylo JA, Wang A, Loftus P, Evans KH, Chu I, Shieh L. Smarter hospital communication: secure smartphone text messaging improves provider satisfaction and perception of efficacy, workflow. J Hosp Med 2014;9(09):573-578

12 Hansen JE, Lazow M, Hagedorn PA. Reducing interdisciplinary communication failures through secure text messaging: a quality improvement project. Pediatr Qual Saf 2018;3(01):e053

13 Patel N, Siegler JE, Stromberg N, Ravitz N, Hanson CW. Perfect storm of inpatient communication needs and an innovative solution utilizing smartphones and secured messaging. Appl Clin Inform 2016;7(03):777-789

14 Moriates C, Wong BM. High-value care programmes from the bottom-up... and the top-down. BMJ Qual Saf 2016;25(11):821-823

15 Ogunlayi F, Britton P. Achieving a 'top-down' change agenda by driving and supporting a collaborative 'bottom-up' process: case study of a large-scale enhanced recovery programme. BMJ Open Qual 2017;6(02):e000008

16 Haun JN, Hathaway W, Chavez M, et al. Clinical practice informs secure messaging benefits and best practices. Appl Clin Inform 2017;8(04):1003-1011 